\title{
Uso de Mapas Paramétricos como auxílio a Gestão e Planejamento Urbano'
}

\section{Parametric Maps as a tool for Urban Management and Planning}

\section{Almeida, Caio Augusto Rabite de Almeida'; Brandão, Guilherme Valle Loures2; Amaral, Wilian Daniel Henriques do3; Silva, Eduardo Lima ${ }^{4}$}

\author{
1 Universidade Federal de Minas Gerais, Av. Barão do Rio Branco, 2872/710 - \\ Juiz de Fora, Brasil, caioaugusto.ara@gmail.com \\ 2 Faculdade do Sudeste Mineiro, guilherme.loures@engenharia.ufjf.br \\ 3 Instituto Federal de Educação, Ciência e Tecnologia do Sudeste de Minas \\ Gerais, wilian.amaral@engenharia.ufjf.br \\ ${ }^{4}$ Universidade Federal de Juiz de Fora, eduardo.lima@arquitetura.ufjf.br
}

\begin{abstract}
RESUMO
Existe, atualmente, uma tendência progressiva de incorporar estratégias de design paramétrico no planejamento urbano que pode representar a distribuição espacial de parâmetros de desenvolvimento em determinada área selecionada. O objetivo do trabalho é apresentar a pesquisa realizada com metodologia de planejamento e regulação do desenvolvimento, baseada em mapeamentos e condicionantes paramétricos. Os mapas paramétricos representam o espaço diretamente em ambientes de ferramentas digitais, permitindo assim que várias alternativas possam ser avaliadas e simuladas, contribuindo para questões como automação urbana e urbanismo informacional. Através de um estudo de caso para uma proposição de análise e simulação de uma área urbana na cidade de Juiz de Fora, utilizando-se de uma situação controle para efeitos de possíveis aumentos de densidade e crescimento urbano. Os resultados permitiram entender as relações possíveis na geração de soluções, em um cenário digital apoiado em ferramentas de natureza paramétrica, que auxiliem os profissionais ligados ao planejamento urbano, somando-se aos mecanismos tradicionais de regulamentação e diretivas. A principal contribuição deste trabalho foi o apontamento das potencialidades e limitações deste sistema de abordagem.
\end{abstract}

Palavras-chave: Urbanismo Paramétrico, Design Urbano, Simulação urbana, Planejamento urbano.

\begin{abstract}
Currently there is a progressive tendency to incorporate parametric design strategies into urban planning, which may represent the spatial distribution of development parameters across a selected area. This work has as objective to present a research carried out with a methodology of planning and development regulation based on parametric mapping and

${ }^{1}$ ALMEIDA, Caio Augusto Rabite de Almeida; BRANDÃO, Guilherme Valle Loures; AMARAL, Wilian Daniel Henriques do; SILVA, Eduardo Lima. Uso de Mapas Paramétricos como auxílio a Gestão e Planejamento Urbano. In: II SIMPÓSIO NACIONAL DE GESTÃO E ENGENHARIA URBANA: SINGEURB, 2019, São Paulo. Anais... Porto Alegre: ANTAC, 2019.
\end{abstract}


conditioners. These parametric maps perform space solutions 'directly within digital tools, thus allowing several alternatives to be evaluated and created, contributing to issues such as urban automation and informational urbanism. The suggested method had as a case study a proposal of an analysis and simulation of an urban area in the city of Juiz de Fora with the type of digital approach in counterpoint to traditional, using a control situation for the effects of possible increases in density and urban growth. The results allowed us to understand the relationships that may be possible to be applied in a digital scenario supported by parametric tools for the generation of solutions that help the professionals involved in urban planning and the contribution with traditional regulatory mechanisms and directives. The main contribution of this work is pointing out the potentialities and limitations of this approach system.

Keywords: Parametric Urbanism, Urban Design, Urban Simulation, Urban Planning.

\section{INTRODUÇÃO}

Mudanças ambientais e avanços tecnológicos, cada vez mais frequentes, modificam as relações no espaço urbano e levam à necessidade de adaptação e reorganização das cidades em diversos níveis. Ao passo em que as cidades contemporâneas se tornam cada vez mais dinâmicas, complexas e abertas para alterações futuras, os instrumentos de operação e regulamentação tradicionais do desenvolvimento urbano, como por exemplo planos diretores e leis orgânicas, passam a ser mais contestados (VIDMAR; KOZELJ, 2015; BEIRÃO, 2012).

A crítica ao planejamento tradicional baseia-se, principalmente, na inflexibilidade que leva à incapacidade de promover mais rapidamente as respostas necessárias, acompanhando o ritmo das mudanças e a imprevisibilidade destas transformações. O tempo e o custo geralmente necessários para discussão e reformulação dos planos diretores também podem ser considerados como fatores de crítica (VIDMAR; KOZELJ; 2015).

Schnabel e Karakiewicz (2009, p. 94) apontam que os planos diretores são em geral prescritivos e têm a intenção de serem precisos, não abertos a mudanças e variações necessárias e inevitáveis, sem atender à rapidez do desenvolvimento informal e flexibilização emergentes. Vidmar e Koželj $(2015$, p. 45) defendem que a forma dos planos diretores tradicionais se tornou rígida demais para lidar com as incertezas e dinâmicas das cidades atuais. Para Billen et al (2014, p.72) é preciso encontrar novos paradigmas de modelagem da forma urbana, de tipos fixos e pré determinações de elementos do modelo para a introdução de conceitos que sejam tão generativos em forma quanto analíticos.

Diversos sistemas e estratégias de planejamento para mitigação destes efeitos de imprevisibilidade vem sendo propostos como meios de planejamento alternativos, como o planejamento baseado em desempenho ou códigos com base em formulários, que começou a surgir na última metade do século XX (HIRT, 2007). Esses questionamentos e a aspiração pelo desenvolvimento de novas técnicas e métodos de planejamento e design urbano com respostas mais rápidas e previsíveis, tanto no espaço quanto em tempo, tornamse, então, prementes no planejamento das cidades (DUARTE, 2011).

\section{PLANEJAMENTO URBANO TRADICIONAL, PLANOS DIRETORES E URBANISMO PARAMÉTRICO}

Os planos diretores são tradicionalmente definidos pelo planejamento denominado "de cima para baixo", com utilização de mapas 2D e documentos de texto (PISSOURIOUS, 2014). Após a definição de uma série de regulamentos de desenvolvimento, são criadas condições que determinam um espaço de solução que atenda àquele determinado critério de desenvolvimento e projeto, com a finalidade de assegurar a coerência com o tecido urbano baseado neste conjunto de restrições.

Em contrapartida ao planejamento tradicional, o design urbano baseado em modelagem tridimensional paramétrica realiza uma abordagem "de baixo para cima", na medida em que molda o espaço urbano real. Realizado o espaçamento e a distribuição dos prédios, os 
efeitos de forma e espaço preexistentes são observados e avaliados, tornando o processo de design deste tecido mais próximo da realidade e gerando alternativas mais dinâmicas das transformações das cidades.

Para Steinø et al. (2005, p.1) a introdução do desenho computacional no urbanismo aumenta seu potencial de sucesso ao parametrizar aspectos como uso, forma, densidade, espaço e tipologia. Verebes (2013) defende que, em vez de se projetar uma solução final, como um plano diretor, deve-se priorizar o planejamento de um sistema de controle que permita à solução evoluir gradualmente com o tempo.

Ainda que em um primeiro momento essas abordagens pareçam antagônicas e distintas, podem estabelecer diálogos complementares ao tratar do planejamento tanto por um viés objetivo e quantificável, quanto por critérios específicos e subjetivamente qualitativos. A dificuldade em aplicar efetivamente essas abordagens qualitativas e quantitativas em planos diretores gera rigidez de planejamento, justificando o uso de mapas paramétricos, otimizando o plano diretor convencional para se tornar um instrumento mais interativo e dinâmico, reagindo melhor às futuras transformações urbanas.

\section{APLICAÇÃO DO MÉTODO}

Um mapa paramétrico é a representação da distribuição espacial dos parâmetros de desenvolvimento em uma área delimitada, atuando como um sistema que regula as propriedades de ordenamento desse espaço (DIVJAK, 1999). Partindo de uma lógica de pensamento paramétrica, podemos observar o desenho urbano como método de modelagem do desenvolvimento usando os valores de meta almejados.

Nessa lógica, pode-se dizer que um edifício definido pelas dimensões de 20×20×30 também pode ser caracterizado como um edifício de 7 pavimentos com área construída de $400 \mathrm{~m}^{2}$ por pavimento (Figura la). Esse processo traz a exploração de múltiplas possibilidades diretamente dentro de um resultado pretendido, já que não se faz necessário o cálculo das dimensões externas do edifício para se atingir os valores finais requisitados (figura lb).

Figura 1 - a) Parametrização do Dimensionamento - relações entre abordagem métrica e paramétrica.

b) Diferentes formas com os mesmos atributos (altura, área)

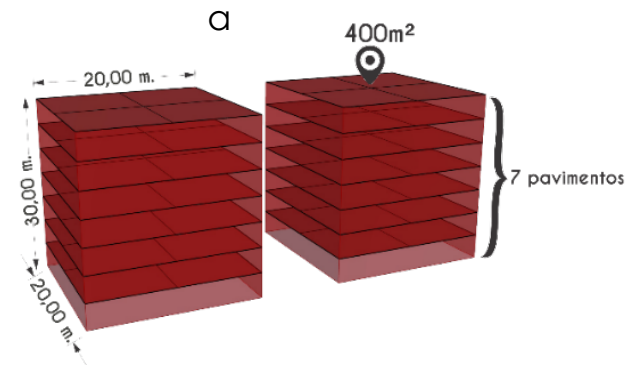

$b$

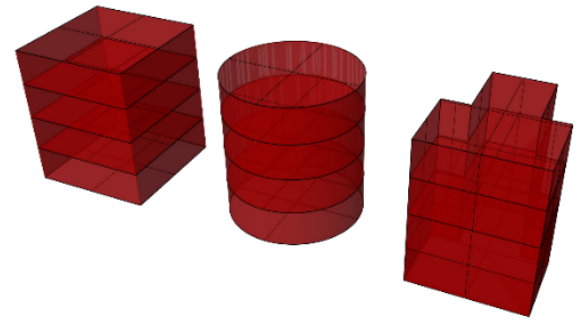

Fonte: Os autores

O critério de seleção da área para a simulação do modelo de planejamento foi a disponibilidade de estudos e análises de configurações por mapas e dados da atual discussão do plano diretor de desenvolvimento urbano vigente: Mapa de Zonas de Especial Interesse Social e Econômico (Fig.2), Mapa de Estruturação e Transformação urbana com a delimitação de seus eixos estruturantes (Fig.3) por serem áreas periféricas, geralmente resultantes de ocupação irregular e ou não planejada.

Os mapeamentos foram organizados em camadas e sobrepostos à base cartográfica em CAD disponível, escolhendo-se então a região de São Pedro para realização da simulação por atender a todas as características de seleção e por ser um dos maiores vetores de crescimento atual. 
Figura 2 - Mapa de Zonas de Especial Interesse Social e Econômico da cidade de Juiz de Fora.

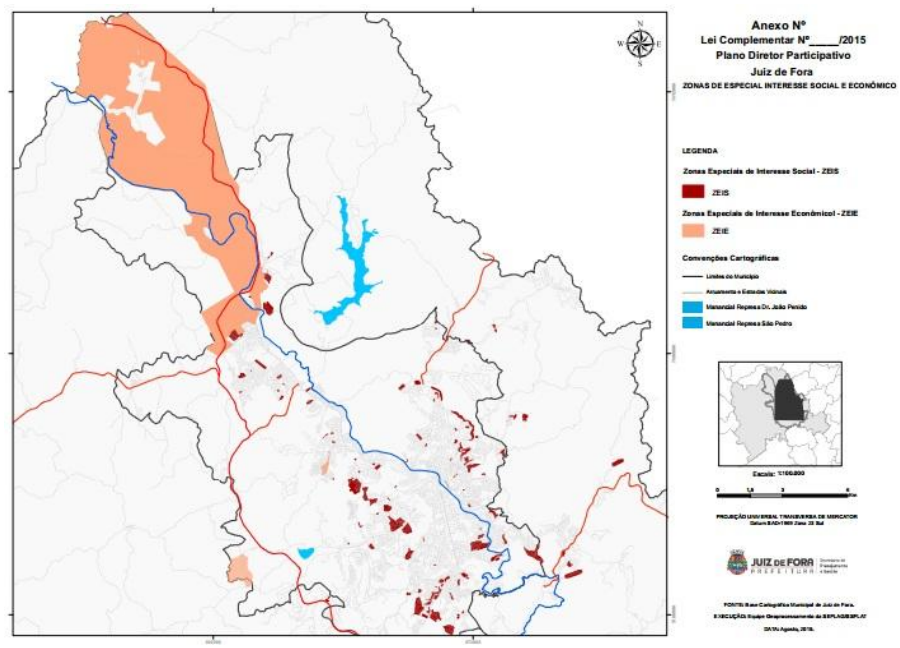

Fonte: Prefeitura de Juiz de Fora. Disponível em: http://www.planodiretorparticipativo.pjf.mg.gov.br/

Figura 3 - Mapa de Estruturação e Transformação Urbana e seus eixos estruturantes da cidade de Juiz de Fora.

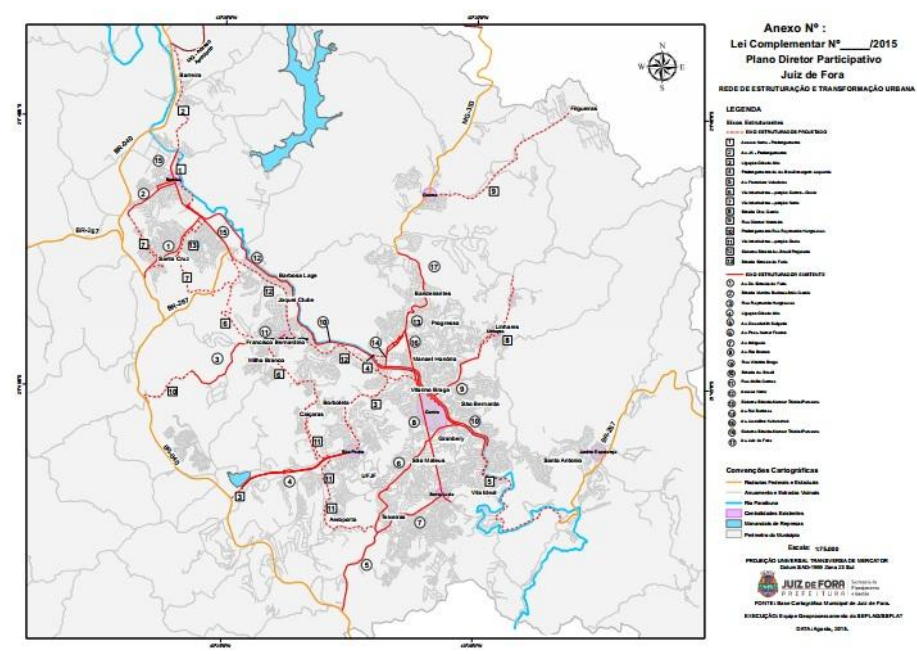

Fonte: Prefeitura de Juiz de Fora. Disponível em: http://www.planodiretorparticipativo.pjf.mg.gov.br/

Utilizando-se de protótipo modelado no software Rhinoceros $\AA^{\circledR}$ com o plugin de programação visual algorítmica Grasshopper $\AA^{\circledR}$, o algoritmo descrito na interface do programa, seguiv estes passos: a)escolheu-se a implantação e a massa do prédio gerada com o número de andares; b) modelou-se o desenvolvimento ou tipo de edifício adaptando-se automaticamente aos parâmetros definidos de acordo com sua localização, ou inseriu-se o edifício na área desejada, com a definição realizando a adaptação de seu volume baseado nos parâmetros definidos para a área.

É importante observar que nem todos os parâmetros são aplicados da mesma forma, pois regulam e controlam o desenvolvimento em níveis diferentes: esses diferentes parâmetros estruturam o código realizado dentro da interface do Grasshopper® (Figura 4) para facilitar o fluxo de dados de entrada e saída. Os parâmetros diretos são usados para definir a forma do desenvolvimento e os indiretos para verificar a possibilidade deste desenvolvimento.

Foram classificados como parâmetros básicos e diretos a serem introduzidos no processo de controle da forma: altura da edificação, número de andares, taxa de ocupação, coeficiente de aproveitamento, volume da construção, área bruta e número de vagas necessárias para estacionamento. Como parâmetro indireto, o tipo máximo permitido do modelo de 
ocupação baseado em determinado zoneamento de acordo com as especificações da legislação urbana da cidade de Juiz de Fora.

Figura 4 - Definição do modelo.

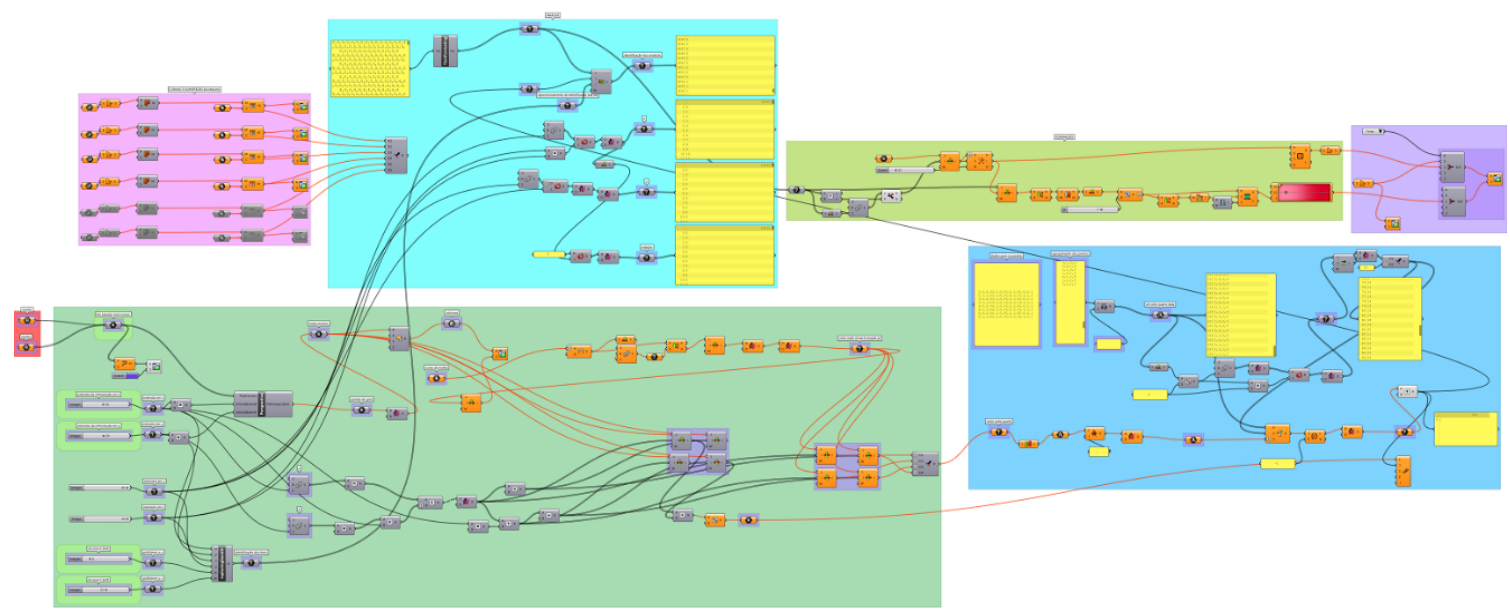

Fonte: Os autores

A simulação foi realizada em um recorte da região tendo como principal foco desenvolver a análise de transformações geradas pela alteração dos parâmetros previamente estabelecidos (Figuras 5 e 6), com alteração dos valores dos coeficientes de aproveitamento, testando diferentes cenários.

Figura 5 - Modelo obtido com a simulação de ocupação com coeficiente de aproveitamento máximo de 2.0.

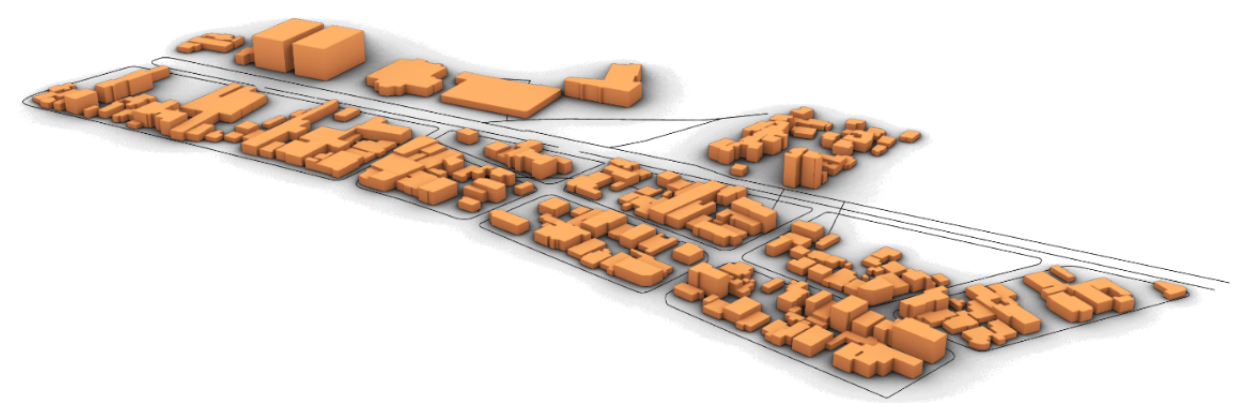

Fonte: Os autores

Figura 6 - Modelo obtido com a simulação de ocupação com coeficiente de aproveitamento máximo de 4.0.

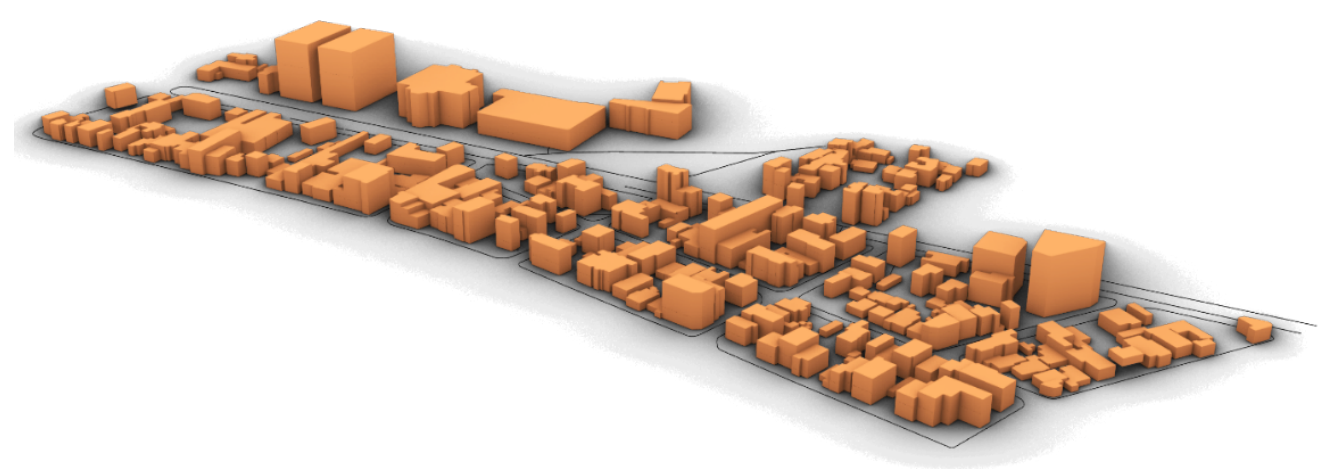

Fonte: Os autores 
O conjunto de parâmetros de controle urbano, como a área do espaço ou o número de estacionamentos necessários é calculado em tempo real e os requisitos são direcionados para o cálculo de parâmetros indiretos de controle, como parte da especificação do uso do solo e zoneamento, facilitando a supervisão de todo o desenvolvimento e focando no design urbano baseado em desempenho.

Parâmetros de regulação indireta são usados para especificar critérios de desempenho (valores de meta) de toda a área de desenvolvimento, unidades espaciais e parcelas individuais de construção, como a área útil permitida ou a distância mínima entre edifícios. Parâmetros indiretos podem ser tipicamente definidos para toda a área de desenvolvimento ou separadamente para cada bloco ou subdivisão urbana do empreendimento.

Embora essas regulamentações não sejam usadas para definir diretamente os volumes do edifício, precisam ser especificadas com antecedência à medida que determinam as restrições do espaço da solução. A ferramenta que usa esse método deve, portanto, calcular automaticamente os valores de controle urbano e verificar se as restrições estão sendo seguidas, permitindo que os projetistas trabalhem em consonância com a regulamentação.

\section{RESULTADOS}

A aplicação demonstrou que o uso de mapas paramétricos junto aos mecanismos tradicionais de controle urbano aprimora e contribui para o processo de projeto urbano, potencializado pela velocidade e flexibilidade da ferramenta que possibilita verificar muitas propostas e alternativas de desenvolvimento. A maior contribuição desse método é não definir apenas o estado final do desenvolvimento, mas as regras interativas com que este desenvolvimento se direciona.

Observa-se como principais resultados e benefícios do uso de ferramentas paramétricas:

- Design rápido ao traçar diferentes propostas de desenvolvimento;

- Flexibilidade e rapidez na geração de alternativas baseadas em diversas condições;

- Avaliação rápida sobre o desenvolvimento de determinada área;

- Tomadas de decisão bem informadas pelo uso de programação visual e visualização das alterações com feedback em tempo real;

Alguns entraves foram detectados, como:

- A elaboração dos mapas pode ser demorada;

- Problemas de comunicação, já que os planejadores urbanos podem não ser programadores ou familiarizados com ferramentas de design paramétrico;

- Erros em mapas paramétricos de grande extensão são de difícil detecção;

- Pode ocorrer redundância dos mapas ao se criar desenvolvimentos de pequena escala.

\section{CONCLUSÕES}

A forma urbana da cidade contemporânea é reflexo da complexidade dos diversos processos que não são acompanhados pela gestão territorial, produzindo espaços fragmentados e de baixa qualidade, responsáveis por acentuar conflitos espaciais, socioeconômicos e ambientais progressivamente. Essas rápidas alterações dentro do ecossistema urbano, têm colocado em xeque os métodos tradicionais de planejamento e regulação do desenvolvimento, demandando cada vez mais um planejamento urbano adaptável e responsivo.

Através das simulações do estudo de caso verifica-se que os mapas paramétricos podem auxiliar os instrumentos tradicionais de regulação e planejamento como por exemplo planos diretores, que se desenvolvidos com a maior entrada e disponibilidade possível de dados e parâmetros, tendem a fornecer resultados e respostas que atendam melhor as necessidades 
da cidade contemporânea. Se comparado a outros métodos emergentes de planejamento urbano, o uso de mapas e ferramentas baseadas em uma lógica paramétrica é capaz de fornecer uma estrutura geral intuitiva de utilização simplificada, favorecendo a compreensão de diversos cenários de transformação espacial.

Por fim, nota-se que o desafio é gerar aos administradores e profissionais relacionados à gestão das cidades informações apropriadas e atualizadas para a tomada de decisões proativas e que permitam um desenvolvimento sustentável de maneira mais efetiva, no entanto o que se observa no contexto da maioria das grandes cidades brasileiras é a inexistência de um sistema coordenado de gestão da informação que possa apoiar outros métodos de gerenciamento urbano, aliada ao fato de que uma grande proporção do crescimento ocorre fora do processo formal de gestão e administração, não alimentando o intenso fluxo de informações através dessas ferramentas emergentes de projeto e simulação urbana.

\section{AGRADECIMENTOS}

Agradecemos à CAPES pelo apoio concedido no desenvolvimento desta pesquisa.

\section{REFERÊNCIAS}

BEIRÃO, J. N. Designing Grammars for Urban Design. A+BE | Architecture and the Built Environment (Vol. 2). Delft: TU Delft, 2012. Doi: http://dx.doi.org/10.7480/a+be.vol2.diss5

BILLEN, R., CUTTING-DECELLE, A.-F., MARINA, O., DE ALMEIDA, J.-P., CAGLIONI, M., FALQUET, G., "... ZLATANOVA, S. 3D City Models and urban information: Current issues and perspectives. In: BILLEN, R., CUTTING-DECELLE, A.-F., MARINA, O., DE ALMEIDA, J.-P., CAGLIONI, M., FALQUET, G., "... ZLATANOVA, S. (EDS.). 3D City Models and urban information: Current issues and perspectives - European COST Action TU0801. France: EDP Sciences, 2014. Doi: http://dx.doi.org/10.1051/TU0801/201400001.

DIVJAK, M. Guiding urban development on the municipal level. Urbani izziv, 10(2), 163-167. 1999. http://dx.doi.org/10.5379/urbani-izziv-1999-10-02-002.

DUARTE, J.P. Customising Mass Housing: A Discursive Grammar for Siza's Malagueira Houses. Ph.D. Dissertation, MIT. 2011.

HIRT, S. The Devil Is in the Definitions. Journal of the American Planning Association, 73(4), $436-$ 450. 2007. Doi: http://dx.doi.org/10.1080/01944360708978524.

PISSOURIOS, I. A. Top-down and bottom-up urban and regional planning: towards a framework for the use of planning standards. European Spatial Research and Policy, 21 (1), 83-99. 2014. http://dx.doi.org/10.2478/ esrp-2014-0007.

SCHNABEL, M. A.; KARAKIEWICZ, J. Rethinking Parameters in Urban Design. International Journal of Architectural Computing, 5(1), 84-98. 2009. Doi:

http://dx.doi.org/10.1260/147807707780912994.

STEIN $\varnothing$, N. VEIRUM, N. Parametric Urban Design. In AESOP 05 Congress, 13-17 Júlio, 2005, Viena. Anais.

VEREBES, T. Masterplanning the Adaptive City: Computational Urbanism in the Twenty-First Century. Nova York: Routledge, 2013.

VIDMAR, J.; KOŽELJ, J. Adaptive urbanism: a parametric maps approach. In: teorija in praksa urejanja prostora | THE CREATIVITY GAME - Theory and Practice of Spatial Planning (p. 44-52). 2015. http://dx.doi.org/10.15292/IU-CG.2015.03.044-052. 